\title{
Superregular Breathers in Optics and Hydrodynamics: Omnipresent Modulation Instability beyond Simple Periodicity
}

\author{
B. Kibler, ${ }^{1, *}$ A. Chabchoub, ${ }^{2}$ A. Gelash, ${ }^{3}$ N. Akhmediev, ${ }^{4}$ and V. E. Zakharov ${ }^{3,5,6}$ \\ ${ }^{1}$ Laboratoire Interdisciplinaire Carnot de Bourgogne (ICB), \\ UMR 6303 CNRS-Université de Bourgogne Franche-Comté, 21078 Dijon, France \\ ${ }^{2}$ Department of Ocean Technology Policy and Environment, Graduate School of Frontier Sciences, \\ The University of Tokyo, Kashiwa, Chiba 277-8563, Japan \\ ${ }^{3}$ Novosibirsk State University, Novosibirsk 630090, Russia \\ ${ }^{4}$ Optical Sciences Group, Research School of Physics and Engineering, \\ The Australian National University, Canberra, ACT 2600, Australia \\ ${ }^{5}$ Lebedev Physical Institute, Russian Academy of Sciences, Moscow 119991, Russia \\ ${ }^{6}$ University of Arizona, Tucson, Arizona 857201, USA
}

(Received 25 June 2015; revised manuscript received 11 September 2015; published 13 November 2015)

\begin{abstract}
Since the 1960s, the Benjamin-Feir (or modulation) instability (MI) has been considered as the selfmodulation of the continuous "envelope waves" with respect to small periodic perturbations that precedes the emergence of highly localized wave structures. Nowadays, the universal nature of MI is established through numerous observations in physics. However, even now, 50 years later, more practical but complex forms of this old physical phenomenon at the frontier of nonlinear wave theory have still not been revealed (i.e., when perturbations beyond simple harmonic are involved). Here, we report the evidence of the broadest class of creation and annihilation dynamics of MI, also called superregular breathers. Observations are done in two different branches of wave physics, namely, in optics and hydrodynamics. Based on the common framework of the nonlinear Schrödinger equation, this multidisciplinary approach proves universality and reversibility of nonlinear wave formations from localized perturbations for drastically different spatial and temporal scales.
\end{abstract}

DOI: 10.1103/PhysRevX.5.041026

\section{INTRODUCTION}

Nonlinearity plays a fundamental role both in the understanding of most natural phenomena and in the development of advanced technologies and engineering designs. The nonlinear Schrödinger equation (NLSE) in its many forms serves as a central model in nonlinear science, and more specifically in nonlinear wave theory [1]. In particular, the one-dimensional NLSE captures the physics of some very exciting and fascinating nonlinear phenomena, such as the modulation instability and envelope solitons [2-3]. It also belongs to the remarkable class of integrable systems [4] and can be solved by using the inverse scattering transform method or other integration techniques. Studies of the phenomenon of modulation instability (MI) have been pioneered by the theoretical and experimental observations done in the 1960s [5-10].

\footnotetext{
*Corresponding author. bertrand.kibler@u-bourgogne.fr

Published by the American Physical Society under the terms of the Creative Commons Attribution 3.0 License. Further distribution of this work must maintain attribution to the author(s) and the published article's title, journal citation, and DOI.
}

Subject Areas: Fluid Dynamics, Nonlinear Dynamics, Optics

The effect has been understood as an instability of a plane wave against the long-wave modulation and associated with the growth of spectral sidebands. MI has been found in deep water $[9,10]$, in plasmas [11-13], within laser light $[8,14]$, in electrical transmission lines [15], and in Bose-Einstein condensates [16]. A linear stability analysis is typically performed to identify the instability criterion and to evaluate the initial growth rate of the sidebands. However, the above simplistic analysis provides only snapshots of the initial steps of MI and the complete evolution of the process has remained unexplored. In reality, MI exhibits much richer dynamics when one goes beyond the simple linear stability analysis. To address this problem, some exact pulsating solutions (also called breathers) describing the full MI dynamics were derived during the 1970s and 1980s, but only for periodic initial modulations [17-22]. These cases provided, as a first step, a powerful framework for interpretation of a specific range of MI-related dynamics that play a fundamental role in the theory of freak waves [23]. Only recently, some unique features of these solutions have been confirmed experimentally for various nonlinear media [24-31]. It concerns the simplest solutions that are either periodic in space and localized in time or periodic in time and localized in 
space; they are referred to as Kuznetsov-Ma breathers and Akhmediev breathers, respectively. Taking the period of both solutions to infinity gives rise to a first-order doubly localized Peregrine soliton. Yet, despite the worldwide success, in particular, stimulating new theoretical studies on the rich family of breather solutions (see Ref. [32] and references therein), theoretical or experimental results are restricted so far to an essentially reduced class of breathers.

In 2013, a new step was taken in theory. Namely, Zakharov and Gelash proposed superregular breather solutions to describe a novel and general scenario of modulation instability that develops from localized perturbations of the plane wave $[33,34]$. The latter have more physical and practical significance than periodic perturbations in Benjamin-Feir instability that require the whole infinite space. Superregular breathers exhibit a combination of unique features in terms of propagation behavior. In contrast to previous analytical solutions, they start with infinitesimally small localized perturbations. Indeed, Akhmediev breathers are known to start the MI from small periodic perturbation. They are fully described by the heteroclinic trajectory in the phase space (adding a phase shift with propagation), whereas Kuznetsov-Ma breathers show pulsating dynamics, starting the propagation from a solitary envelope. Superregular breathers form a subset of breather solutions and are defined as a nonlinear superposition of $N$ pairs (where $N$ is an integer) of quasiAkhmediev breathers propagating in opposite directions. Most interestingly, superregular breathers may describe nonlinear dynamics of the broadest class of unstable localized small perturbation of the plane wave, thus providing a novel and global overview of the nonlinear stage of modulation instability. They can be considered as the fundamental "building blocks" for the complete description of the modulation instability and the old problem of integrable turbulence, and even the so-called freak waves induced by MI for coherent or incoherent wave trains [35]. In addition to the development of perturbation into special pairs of breathers, the reverse process is another important scenario. Indeed, $N$ pairs of quasi-Akhmediev breathers almost annihilate (we call this process "quasiannihilation") to a small localized perturbation as a result of their collision $[33,34]$. Such mathematical entities (i.e., $N$-pair breather solutions with their shifting parameters) are able to describe different configurations of the nonlinear stage of MI at any propagation distance, such as the amplification, annihilation, or even ghost interaction of nearly any individual or ensemble of localized perturbations (i.e., local concentration of energy) on a finite background (as revealed later by the special cases of the one-pair breather solution shown in Fig. 1). In practice, most of spontaneous pattern formations and localized structures induced by MI could now be described by using such generalized solutions, even when the background itself is localized in time (i.e., a laser pulse). It was already shown that breather dynamics with pulsed excitation can be interpreted in terms of local breather states at different points on the pulse envelope [36,37]. Superregular breathers give an enlarged and generalized picture of the collision features with respect to recent numerical and experimental studies of breather structures and their interactions in optics or hydrodynamics [29-31,38].

In this work, we simultaneously report two experiments, in an optical fiber and in a water-wave tank, specifically designed to reveal the corresponding dynamics of such mathematical entities as superregular breathers in real physical systems. We demonstrate explicitly that initial perturbation develops to a superregular pair of quasi-Akhmediev breathers that move in opposite directions leaving behind a nonperturbed plane wave. We also observe the reverse process of annihilation of a pair of quasi-Akhmediev breathers. Our results are in excellent agreement with analytic predictions. Moreover, our multidisciplinary approach confirms the universal nature of superregular breathers for any NLSE-driven wave system through comprehensive analysis of unstable localized perturbations on a time scale of seconds for water waves and on a time scale of picoseconds for light waves.

\section{BREATHER FORMALISM}

Our experiments are designed using the breather formalism of Refs. [33,34] obtained within the framework of the self-focusing NLSE in dimensionless form, $i \psi_{\xi}+1 / 2 \psi_{\tau \tau}+|\psi|^{2} \psi=0$, where subscripted variables stand for partial differentiations. Here, $\psi$ is a wave group or wave envelope which is a function of $\xi$ (a scaled propagation distance or longitudinal variable) and $\tau$ (a comoving time, or transverse variable, moving with the wave-group velocity). This equation usually describes the nonlinear wave evolution in various media, in particular, gravity waves in deep-water conditions and light waves in standard optical fibers. The above evolution equation that describes wave propagation in space (not in time) is more convenient for direct comparison with laboratory experiments.

We study MI of the plane wave solution, which can be written in our parametrization as $\psi_{0}=A e^{i A^{2} \xi}$, where $A$ is the amplitude of the plane wave (here equal to 1 ). The self-focusing NLSE has an exact $N$-breather solution, which can be constructed by the appropriate integration technique (see Appendix A). The general one-breather solution has four real parameters, $R, \alpha, \mu, \theta$, and can be described by the following expression [39-41]: 

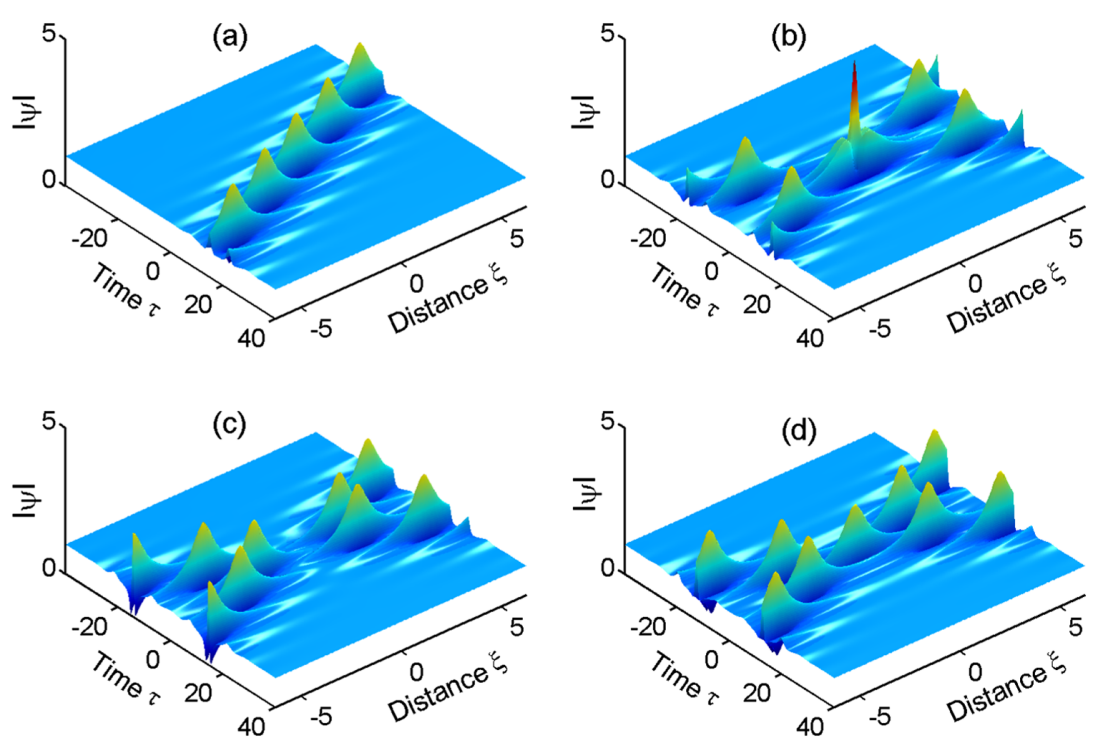

FIG. 1. Special cases of the one-pair breather solution $(R=1.15, \alpha=0.4)$ compared to the single breather solution. (a) Single breather solution $(R=1.15, \alpha=0.4, \theta=0, \mu=0)$. (b)-(d) One-pair breather solution with the following phase shifts: $\theta_{1,2}=0$, $\theta_{1,2}=\pi / 2$ (superregular case) and $\theta_{1,2}=\pi$, respectively $\left(\mu_{1,2}=0\right.$ for all cases). Note that the maximum amplitudes at the origin ( $\xi=0, \tau=0$ ) are (a) 2.86, (b) 4.72, (c) 1.125, (d) 2.72. These values highlight the degree of breather interaction.

$\psi=\left(1+2\left(R+\frac{1}{R}\right) \cos \alpha \frac{q_{1}^{*} q_{2}}{\left|q_{1}\right|^{2}+\left|q_{2}\right|^{2}}\right) e^{i \xi}$,

$q_{1}=e^{-\phi}-\frac{e^{\phi-i \alpha}}{R}, \quad q_{2}=e^{\phi}-\frac{e^{-\phi-i \alpha}}{R}$,

$\phi=\eta \tau+\gamma \xi+\frac{\mu}{2}+i\left(k \tau+\omega \xi-\frac{\theta}{2}\right)$,

$\eta=-\frac{1}{2}\left(R-\frac{1}{R}\right) \cos \alpha, \quad k=-\frac{1}{2}\left(R+\frac{1}{R}\right) \sin \alpha$

$\gamma=-\frac{1}{2}\left(R^{2}+\frac{1}{R^{2}}\right) \sin 2 \alpha, \quad \omega=\frac{1}{2}\left(R^{2}-\frac{1}{R^{2}}\right) \cos 2 \alpha$.

Parameters $R, \alpha$ are the polar coordinates of the point in the area $R \geq 1,(\pi / 2)>\alpha>-(\pi / 2)$ (actually, $R, \alpha$ describe the coordinate of the pole of the solution for the auxiliary Zakharov-Shabat system, see Appendix A). In general, the one-breather solution is a localized object with characteristic size $\delta \tau \sim 1 / 2 \eta=[(R-1 / R) \cos \alpha]^{-1}$, moving on top of the continuous wave with breather group velocity $V_{g}=\gamma / \eta=2 \sin \alpha\left(R^{4}+1\right) /\left[R\left(R^{2}-1\right)\right]$, and oscillating with period $T=2 \pi / 2 \omega=2 \pi R^{2} /$ $\left[\left(R^{4}-1\right) \cos 2 \alpha\right]$ [34]. Thus, $R, \alpha$ control the main breather properties. Parameters $\mu, \theta$ define the location and phase of the breather and vary in the range $[0,2 \pi]$. When $R=1$, $\alpha \neq 0$, the solution is an Akhmediev breather (AB), which becomes the Peregrine soliton in the limit $\alpha \rightarrow 0$. For low values of $V_{g}(R \neq 1, \alpha \rightarrow 0)$, we approach the limit of Kuznetsov-Ma breathers, whereas for high values of $V_{g}$ and $\delta \tau(R=1+\varepsilon$, where $\varepsilon$ is a small parameter), we obtain the class of quasi-Akhmediev breathers, which play the key role in the theory of superregular breathers. Figure 1(a) shows the example of a quasi-Akhmediev breather with parameters ( $R=1.15, \alpha=0.4)$. In the general $N$-breather solution, each $i$ th breather has similar four parameters, $R_{i}, \alpha_{i}$, $\mu_{i}, \theta_{i}$, so that the total solution is described by $4 N$ parameters.

Zakharov and Gelash have found that pairs of quasiAkhmediev breathers with opposite values of angular parameter $\alpha$ form the broadest class of $N$-pair breather solutions that describes the nonlinear stage of MI of small localized perturbation as well as breather quasiannihilation (see Appendix A for a short mathematical comment). The examples of complicated two- and three-pairs scenarios as well as a discussion of general $N$-pairs solutions can be found in Refs. [33,34]. Note that now the limit $N \rightarrow \infty$ is under theoretical study. Here, we consider only the simplest, but very important, case of superregular one-pair solution with parameters appropriate for our experimental setups. The onepair superregular solution can be obtained from a general two-breather solution by setting $R_{1}=R_{2}=R=1+\varepsilon$, $\alpha_{1}=-\alpha_{2}=\alpha$, and can be written in the following form:

$$
\begin{aligned}
\psi= & {\left[1+\left(R^{2}-\frac{1}{R^{2}}\right) \frac{N}{\Delta} \sin 2 \alpha\right] e^{i \xi}, } \\
N= & \left(R-\frac{1}{R}\right) \sin \alpha\left(\left|\boldsymbol{q}_{\mathbf{1}}\right|^{2} q_{21}^{*} q_{22}+\left|\boldsymbol{q}_{\mathbf{2}}\right|^{2} q_{11}^{*} q_{12}\right) \\
& -i\left(R+\frac{1}{R}\right) \cos \alpha\left[\left(\boldsymbol{q}_{\mathbf{1}}^{*} \cdot \boldsymbol{q}_{\mathbf{2}}\right) q_{21}^{*} q_{12}-\left(\boldsymbol{q}_{\mathbf{1}} \cdot \boldsymbol{q}_{\mathbf{2}}^{*}\right) q_{11}^{*} q_{22}\right], \\
\Delta= & \left(R+\frac{1}{R}\right)^{2} \cos ^{2} \alpha\left|q_{11} q_{22}-q_{12} q_{21}\right|^{2} \\
& +\left(R-\frac{1}{R}\right)^{2}\left|\boldsymbol{q}_{\mathbf{1}}\right|^{2}\left|\boldsymbol{q}_{\mathbf{2}}\right|^{2} \sin ^{2} \alpha,
\end{aligned}
$$


where $\boldsymbol{q}_{\boldsymbol{i}}=\left(q_{i 1}, q_{i 2}\right)$ is a two-component vector function, so that

$$
\begin{aligned}
& q_{11}=e^{-\phi_{1}}-\frac{e^{\phi_{1}-i \alpha}}{R}, \quad q_{12}=e^{\phi_{1}}-\frac{e^{-\phi_{1}-i \alpha}}{R}, \\
& q_{21}=e^{-\phi_{2}}-\frac{e^{\phi_{2}+i \alpha}}{R}, \quad q_{22}=e^{\phi_{2}}-\frac{e^{-\phi_{2}+i \alpha}}{R}, \\
& \phi_{1}=\eta \tau+\gamma \xi+\frac{\mu_{1}}{2}+i\left(k \tau+\omega \xi-\frac{\theta_{1}}{2}\right) \\
& \phi_{2}=\eta \tau-\gamma \xi+\frac{\mu_{2}}{2}-i\left(k \tau-\omega \xi+\frac{\theta_{2}}{2}\right)
\end{aligned}
$$

and the parameters $\eta, k, \gamma$, and $\omega$ are defined above. The small parameter $\varepsilon=(R-1)$ characterizes the amplitude of the perturbation at the moment of breather quasiannihilation. Parameters $\mu_{1,2}, \theta_{1,2}$ correspond to the phase shifts between the two breathers; $\mu_{1,2}$ can also describe shifts in space and time (see also Appendix A). Such phase shifts affect the shape and the amplitude of the perturbation. The quasiannihilation appears when $\theta_{1}+\theta_{2}$ is close to $\pi$. The difference between $\theta_{1}+\theta_{2}$ and $\pi$ defines the degree of complexity of the wave profile at the area of collision. The solution is superregular when the phases are adjusted in a way that the perturbation is sufficiently small.

Figures 1(b)-1(d) shows some particular arrangements of the above one-pair breather solution $(R=1.15, \alpha=0.4)$ with the following values of phase shifts: $\theta_{1,2}=0$, $\theta_{1,2}=\pi / 2$, and $\theta_{1,2}=\pi$, respectively $\left(\mu_{1,2}=0\right.$ for all cases). The synchronization of the collision is obtained for $\theta_{1,2}=0$, thus leading to the formation of the high amplitude peak at the origin $(\xi=0, \tau=0)$, as shown in Fig. 1(b) $[40,41]$. A similar synchronized collision of Akhmediev breathers has recently been studied in optics experiments [30], by controlling the phase and velocity differences between the breathers. Figure 1(c) shows the quasiannihilation of breathers at the origin when $\theta_{1,2}=\pi / 2$. This corresponds to a small localized perturbation of the continuous wave on the line $\xi=0$ (superregular case) [33,34]. Another particular interaction is obtained for $\theta_{1,2}=\pi$ [see Fig. 1(d)]. Then, the collision point is just another maximum of either breather solution. Each breather then appears seemingly without influence of the collision process; this particular case is here called a ghost interaction of breathers. Quasi-Akhmediev breathers shown in Fig. 1(a) are periodic in neither time nor space. They are periodic along the line connecting the peak maxima. For the one-pair solution, shown in Figs. 1(b)-1(d), there is only one collision point in the plane $(\xi, \tau)$. After this collision, breathers propagate in opposite directions to infinity with a constant group velocity $V_{g}$.

Below, we focus on the novel scenario of quasiannihilation of breathers. When $\theta_{1}+\theta_{2}$ is exactly equal to $\pi$ [for example, $\theta_{1,2}=\pi / 2$, like in Fig. 1(c)], the quasiannihilation is the most effective. In this case, at the moment of breather collision $(\xi=0)$ the perturbation $\delta \psi$ on the continuous wave (i.e., $\psi=1+\delta \psi$ ) can be approximated by the following formula:

$$
\delta \psi \approx \frac{4 i \varepsilon \cosh (i \alpha) \cos \left(2 \tau \sin \alpha-\frac{\theta_{1}-\theta_{2}}{2}\right)}{\cosh (2 \varepsilon \tau \cos \alpha)},
$$

where $\varepsilon=R-1$. The perturbation is localized in time and can contain many oscillations, but their number decreases with decreasing $\alpha$. The number of oscillations also defines the main perturbation frequency of the wave envelope. Note that $R$ and $\alpha$ also govern the angle that separates quasi-AB
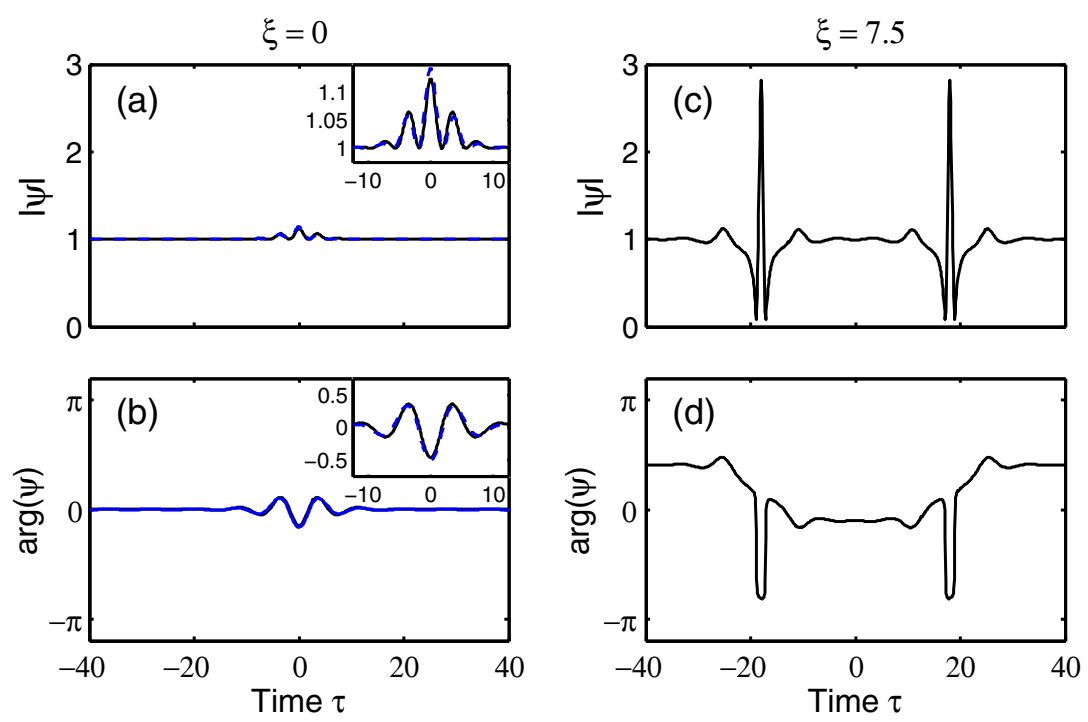

FIG. 2. (a),(b) Amplitude and phase profiles of the approximated perturbation (blue dashed line) calculated with Eq. (2) compared to the exact profile (black solid line) of the quasiannihilated breathers at $\xi=0$ observed in Fig. 1(c). (c),(d) Amplitude and phase profiles of the one-pair breather solution at $\xi=7.5$ with the same parameters as in Fig. 1(c). 
trajectories in the plane $(\xi, \tau)$. Moreover, the temporal width of the initial perturbation increases with decreasing $\varepsilon$, while its amplitude decreases, so $\varepsilon$ plays a role of the small parameter for our perturbation problem. Figures 2(a) and 2(b) show the comparison between the approximated perturbation and the exact profile of the quasiannihilated breathers from Fig. 1(c). We observe that the simplified approach of $\delta \psi$ gives a good fit of the small localized perturbation even here with $\varepsilon=0.15$. In this work, we choose a low value of the parameter $\alpha$ and a considerable value of $\varepsilon$ due to the restrictions of experimental setups. An initially small localized perturbation of the continuous wave grows at first exponentially. It is described by the well-known equations for the linear stage of modulation instability. Then it develops into a pair of breathers propagating in opposite directions, as shown in Figs. 2(c) and 2 (d). We note that such regular solutions leave after the propagation of breathers a continuous wave with a different phase (the difference with the phase at infinity is $4 \alpha$ ) [34]. A process when the perturbed part of the continuous wave almost returns to the initial state can be treated as a local or approximate Fermi-Pasta-Ulam recurrence $[42,43]$. This idea is particularly interesting when we discuss the whole $N$-pair class of superregular breathers, which describes a wide variety of initial perturbations. Note that, for the case of periodic perturbations, the Fermi-PastaUlam recurrence is described by Akhmediev breathers [44] and also leads to the phase shift.

\section{EXPERIMENTAL SETUPS}

The experimental setups used to demonstrate the existence of superregular breathers in real physical systems, i.e., water waves and nonlinear fiber optics, are depicted in Figs. 3(a) and 3(b), respectively. All parameters of our experiments are chosen to highlight the creation and annihilation dynamics related to superregular breathers as shown in Fig. 1(c) (in particular, when $0<\xi<5$ ). Each system is capable of synthesizing nontrivial wave profiles in the temporal domain according to Eqs. (1) and (2). Such an ideal excitation of the modulation

(a) Water-wave tank

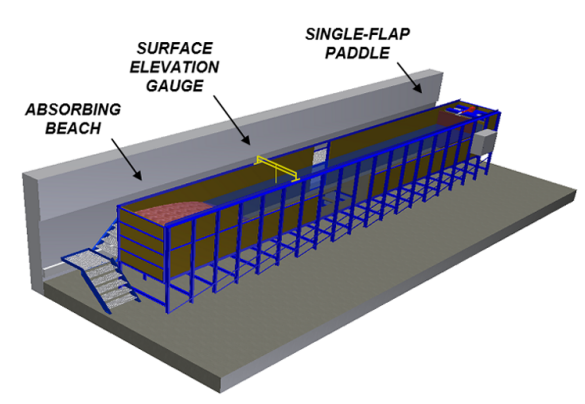

instability process is a prerequisite to exactly observe the dynamics shown in Fig. 1 instead of more complex behaviors [27]. In water-wave experiments, the initial wave profiles are generated with a single-flap paddle located at one end of a tank. An electric signal, calculated using the exact mathematical expression describing the water surface elevation, drives the paddle to directly modulate the surface height in the temporal domain. The tank dimensions are $15 \times 1.6 \times 1.5 \mathrm{~m}^{3}[25,29]$. In order to avoid wave reflections and perform clean experiments in the wave flume, the influence of reflected waves from the beach on the breather dynamics should be minimized as much as possible. Hence, an absorbing beach is installed at the opposite end and we place the last wave gauge $9 \mathrm{~m}$ from the flap. At this position, the beach is at 3-m distance from the wave gauge. Previous experiments, which have been conducted in the same facility, have already shown that at this distance and by choosing small amplitude carrier, the reflective waves do not have any significant influence on the dynamics of NLSE-localized structures [25,29]. To overcome the restrictions related to the finite wave flume length, we split the experiment into several stages and we reconstruct the wave dynamics afterwards [29]. Namely, after starting the breather wave generation repetitively with different initial conditions given from theory, the wave profiles were measured at 4.5 and $9 \mathrm{~m}$ from the flap. Measurements at $9 \mathrm{~m}$ then serve as new initial conditions for the wave maker in the next stage of the experiments. This process is repeated 4 times to reach the propagation distance of $31.5 \mathrm{~m}$. All measurements are then compared to the theoretical predictions.

On the other hand, for light waves, the ideal excitation is obtained through Fourier-transform optical pulse shaping with phase and amplitude controls of the optical field in the spectral domain. This optical processing is based on spectral line-by-line shaping of a frequency comb source and the subsequent amplification stage before coupling into a 0.76 - or $1.5-\mathrm{km}$-long segment of the same standard single-mode optical fiber. After propagation, wave profiles are then simultaneously measured in temporal and spectral domains at the fiber output. The spectral shaping of an

(b) Nonlinear optical fibre workbench

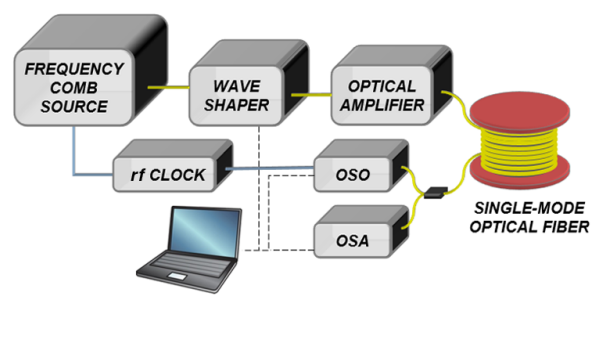

FIG. 3. Experimental setups used to demonstrate the existence of superregular breathers based on (a) a water-wave tank and (b) a nonlinear optical fiber workbench (OSO, optical sampling oscilloscope; OSA, optical spectrum analyzer). 
optical-frequency comb allows us to synthesize tailored modulated initial conditions of the continuous wave through a time-periodic pattern whose frequency is equal to the spectral separation of the comb lines (i.e., here equal to $20 \mathrm{GHz}$ ). We then restrict the theoretical parameters to fit the limited time window over which we can inscribe arbitrary localized perturbation on the continuous envelope wave, but also observe the complete nonlinear dynamics. For further details about the experimental setups, see Appendixes B and C.

\section{RESULTS FOR WATER WAVES}

Figure 4(a) shows the results of experiments carried out in the water-wave tank by considering an initial localized (a) Experiment

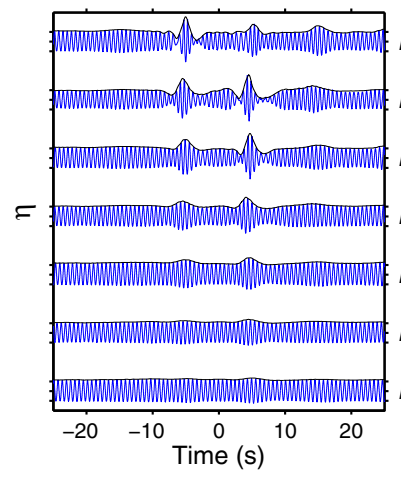

(c) Experiment
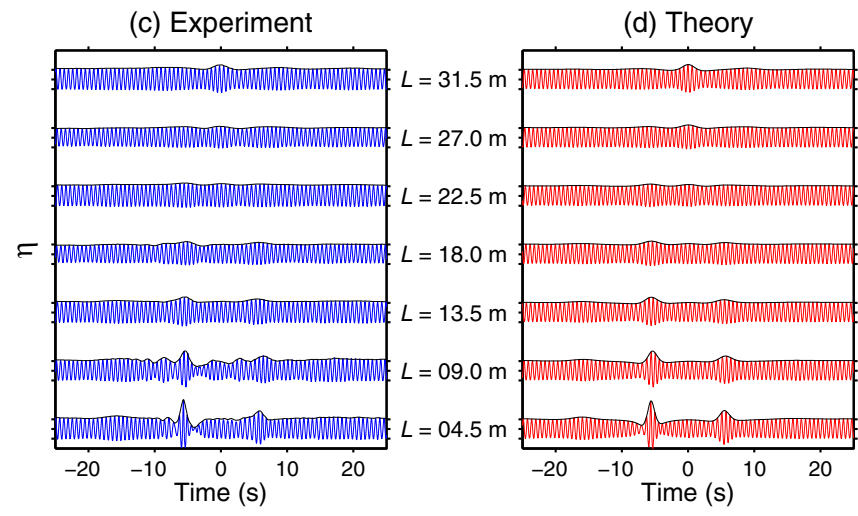

FIG. 4. Experimental water-wave measurements of both creation and annihilation of a pair of breathers compared to theoretical predictions. The initial conditions are generated taking into account the breather propagation along the tank during the excitation. (a),(c) Evolution of time series of surface elevation measurement (blue lines) as a function of propagation distance for creation and annihilation dynamics, respectively. The surface elevation is kept in normalized units so that the amplitude of the unperturbed wave train is 1 (in dimensional units, it corresponds to $1 \mathrm{~cm}$ ). The corresponding envelopes of the wave trains (black lines) are calculated using the Hilbert transform (see Appendix B). (b),(d) Theoretical predictions of time series of surface elevation (red lines) as a function of propagation distance for creation and annihilation dynamics, respectively. The envelope of the analytical solution is shown by black line. perturbation centered at $t=0$, and by using the same parameters as in Figs. 1 and 2. After $18 \mathrm{~m}$ of propagation, we clearly observe that such a perturbation develops into a superregular pair of quasi-Akhmediev breathers that move in opposite directions leaving behind (i.e., at the center $t=0)$ a nonperturbed plane wave. Note that the envelope asymmetry between the two breathers has to be inserted into initial conditions in order to take into account breather propagation along the tank during the excitation. Comparison of the experimental and theoretical results is shown in Fig. 4(b) (see Appendix B). For each time series, we also calculate the envelope profile corresponding to the surface elevation of the wave trains to facilitate the comparison with the theory and to highlight the analogy with the light waves. The comparison between the measured wave profiles and the theoretical curves shows very good agreement for both the amplitude and the phase of the wave trains. Theoretical wave profiles are based on the calculation of the free surface elevation from NLSE solutions and variable $\psi$ given by Eq. (1) to second order in steepness (see Appendix B).

The reverse process implying the quasiannihilation of a pair of quasi-Akhmediev breathers is depicted in Figs. 4(c) and 4(d). Such nonlinear dynamics can be obtained by applying time-reversal operation or reverse propagation, which corresponds to $\theta_{1,2}+\pi$ (i.e., $\xi$ becomes $-\xi$ in Figs. 1 and 2). The parameters of the superregular onepair breather solution are also slightly changed due to experimental restrictions, in particular, shifting parameters (now $\mu_{1}=-2$ and $\mu_{2}=2$, see Appendix A for discussion of the shifting of $\mu_{1,2}$ ). We experimentally confirm here the quasiannihilation dynamics of the initial pair of quasiAkhmediev breathers into a localized perturbation centered at $t=0$, after $31.5 \mathrm{~m}$ of propagation. The agreement with theoretical predictions is again excellent, thus demonstrating the existence of superregular breathers in hydrodynamics, in particular, for describing the nonlinear modulation instability in the framework of localized perturbations. Note that amplitudes are slightly lower in the experiments than theoretical prediction, in particular, due to the dissipation naturally existing when performing experiments in water-wave facilities [45].

\section{RESULTS FOR LIGHT WAVES}

The parameters of the superregular one-pair breather solution are slightly changed $\left(R=1.4, \alpha=0.4, \mu_{1}=-2\right.$, and $\mu_{2}=2$ ) due to the restrictions of the optical workbench used here (i.e., to prevent from any deviation from the NLSE), in particular, with $\varepsilon=0.4$. However, the creation of a pair of breathers propagating in opposite directions as well as their quasiannihilation into a relatively small and localized perturbation of the continuous wave (reversible dynamics) are indeed observed even for a large value of $\varepsilon$, in excellent agreement with the theoretical solutions. All the experimental parameters are embedded into two 
fundamental theoretical parameters $R$ and $\alpha$ defining the breather, which makes the design of such experimental systems very difficult. Here, the use of the static line-byline shaping technique of the optical frequency comb also implies the time-periodic generation of localized perturbations. Future ultrafast optical systems based on a genuine dynamic pulse-shaping technique might allow us to demonstrate the annihilation of successive or isolated localized perturbations with various shapes.

Figure 5 reports the development of a small localized perturbation into a pair of breathers for two propagation distances, when $\theta_{1,2}=\pi / 2$. By comparing Fig. 5(a) with Fig. 5(b), we clearly reveal that each element of the measured periodic wave (i.e., a 50-ps time window) perfectly agrees with theory. Note that no interaction occurs between neighboring elements of the periodic pattern on the studied distance. To complete our experimental characterization in optics since no phase information is

(a) Experiment

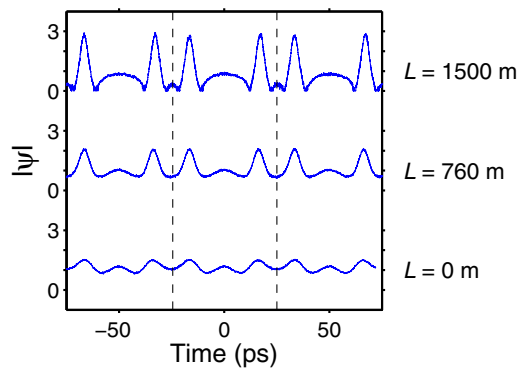

(c)

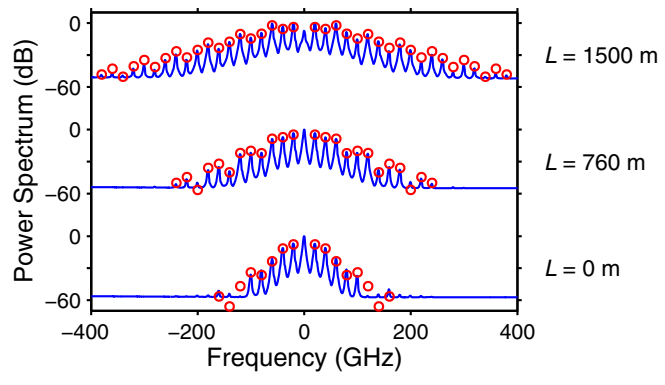

FIG. 5. Experimental light-wave measurements of the creation of a pair of breathers compared to theoretical predictions. (a),(b) Evolution of the square root of intensity profiles in the temporal domain as a function of propagation distance obtained in experiments and corresponding amplitude profiles from theory, respectively. The wave profiles are kept in normalized units so that the amplitude of the unperturbed wave train is 1 . Note that we generate a periodic pattern of the exact solution in our experiments due to the intrinsic features of the frequencycomb-based system; each element of the pattern is delimited by dashed lines. (c) Comparison of the corresponding power spectra (blue solid lines for experiments and red open circles for theory). Note that the theoretical spectra are sampled with the repetition rate of the experimental pattern and the deltafunction component at the pump wavelength (i.e., the continuous envelope wave) is not shown. readily available in the temporal domain, direct spectral measurements of the evolving intensity profile are performed. They are shown in Fig. 5(c). A strong spectral broadening is observed when compared to the initial spectrum of the perturbed continuous wave, thus confirming the nonlinear focusing of perturbations. The excellent signal-to-noise ratio in our experiments also allows the theoretical predictions to be satisfied over a 50-dB dynamic range.

Moreover, we study the reverse process corresponding to the quasiannihilation of a pair of breathers into a small localized perturbation of the continuous wave (i.e., $\left.\theta_{1,2}=\pi / 2+\pi\right)$, as shown in Fig. 6. Here, we confirm the quasiannihilation dynamics after $760 \mathrm{~m}$ of propagation, whereas for longer propagation (i.e., $1500 \mathrm{~m}$ ) we note the emergence of a single localized peak as already shown in the space-time evolution from Fig. 1(c) when looking in the reverse propagation direction. The corresponding spectral signatures are reported in Fig. 6(c). In particular, the quasiannihilation of breathers is associated with significant (a) Experiment

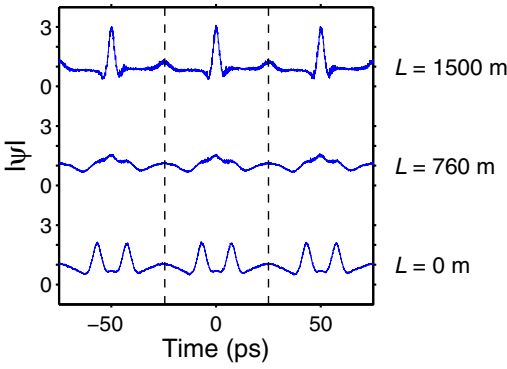

(b) Theory

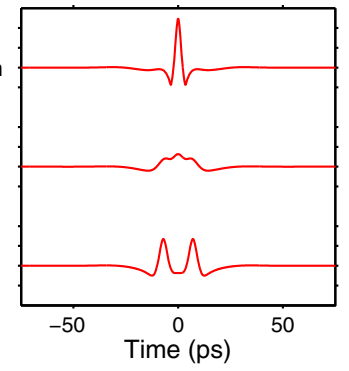

(c)

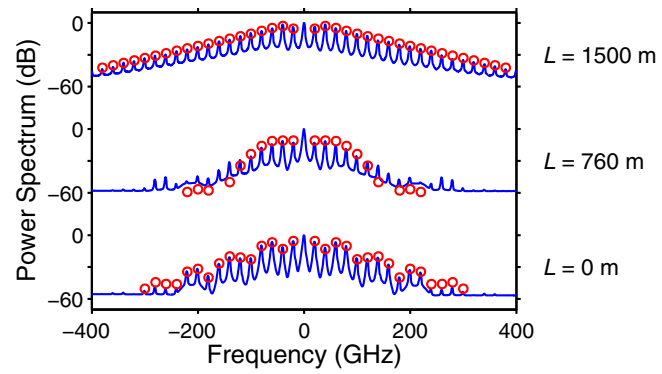

FIG. 6. Experimental light-wave measurements of the quasiannihilation of a pair of breathers compared to theoretical predictions. (a),(b) Evolution of the square root of intensity profiles in the temporal domain as a function of propagation distance obtained in experiments and corresponding amplitude profiles from theory, respectively. Note that we generate a periodic pattern of the exact solution in our experiments due to the intrinsic features of the frequency-comb-based system; each element of the pattern is delimited by dashed lines. (c) Comparison of the corresponding power spectra (blue solid lines for experiments and red open circles for theory). Note that the theoretical spectra are sampled with the repetition rate of the experimental pattern and the delta-function component at the pump wavelength (i.e., the continuous envelope wave) is not shown. 
spectral narrowing. Indeed, the energy distributed in the multiple initial sidebands comes back to the pump and its adjacent first harmonics before being redistributed during the next nonlinear focusing.

\section{DISCUSSION AND CONCLUSION}

From the mathematical point of view, breather solutions of the NLSE are defined as unstable waves. Nonideal conditions will induce the emergence of asymmetric wave profiles or/and introduce some complex spatial recurrence phenomenon. The unstable wave will diverge from the ideal NLSE dynamics during propagation. However, in practice, it is worth mentioning that the main signatures of breather dynamics will persist and be strongly noticeable as reported in previous experimental works in optics and using nonideal conditions. Note that exact breather solutions exist in the case of more complex integrable models such as the Hirota equation, taking into account higher-order terms similar to third-order dispersion, nonlinear dispersion, etc. (see Ref. [46] and the references therein). But, in the presence of cavity boundary conditions and significant dissipation, the issue of modulation instability would be strongly impacted [47]. Even if the modulation instability process is known as a possible common precursor of complex breathing localized structures, the underlying dynamics strongly depends on the dissipative nature of the system. For instance, a continuous wave can be destabilized by weak perturbations leading to the emergence of well-known Turing patterns via modulation instability. But more complex intermittent dynamics with nontrivial periodicity can be observed in distinct configurations of dissipative optical systems [48,49].

The experimental setups used here to demonstrate the existence of superregular breathers in nearly conservative physical systems, i.e., water waves and nonlinear fiber optics, were designed in such a way as to prevent any contribution from higher-order effects beyond the secondorder dispersion and the cubic nonlinearity. In particular, the nature of such additional terms in generalized NLSEs may strongly differ between both systems, thus making the analysis and comparison of experimental results more difficult. In optics, we prefer to avoid the detrimental effects of Raman scattering and third-order dispersion, whereas in hydrodynamics we try to restrain the impact of higher-order dispersion and the mean flow. Our work explicitly confirms the analogy of complex nonlinear dynamics between wave propagation in optical Kerr media and water waves and, therefore, in a general context of weakly nonlinear dispersive media, when the NLSE accurately approximates the governing equation of the media of interest [50]. Note that the main differences between our two experimental approaches lie in only two technical aspects related to substantially different temporal scales of breather waves: (i) the initial shaping of the perturbation on the plane wave and (ii) the measurement of wave profiles.
In water-wave experiments, one directly drives the paddle to modulate the surface height of the water in the temporal domain on a time scale of seconds. In contrast, for light waves, the ultrafast excitation on a time scale of picoseconds can only be obtained through Fourier-transform optical pulse shaping with phase and amplitude controls of the optical field in the spectral domain. For wave measurements that are performed at a fixed point, we obtain both amplitude and phase of the wave trains in the temporal domain for water waves, whereas in optics, we record the intensity profiles of a wave envelope in both time and frequency domains.

In conclusion, we report the first successful experimental evidence of fundamental theoretical "building blocks," called superregular breather waves, that describe the general scenario of modulation instability. In particular, our results show both creation and annihilation of nonlinear oscillatory wave dynamics from or towards small localized perturbations. As a consequence, this also constitutes an experimental proof of time-reversal invariance of the general scenario of modulation instability due to the intrinsic properties of the NLSE. Unlike all previous experimental studies of limiting cases of breather solutions, we investigate here the unexplored class of small localized perturbations related to the broad class of quasibreather solutions and their interaction. In addition, we provide simultaneous demonstrations in two distinct disciplines of wave physics, namely, optics and hydrodynamics. Such a multidisciplinary approach reveals once again the universal character of the modulation instability of continuous envelope waves with respect to small perturbations through different time scale analysis: on a second time scale for water waves and on a picosecond time scale for light waves. We believe that these results represent a major step towards a global understanding of the nonlinear stage of modulation instability, one of the most ubiquitous types of instabilities in nature, as well as a first attempt to solve wave turbulence in integrable systems. It is worth mentioning that our exceptional capability to manipulate light waves and water waves that confirms the frontier of nonlinear wave theory at different physical scales will strongly impact the numerous disciplines where modulation instability plays an important role in pattern formation mechanisms.

\section{ACKNOWLEDGMENTS}

B. K. gratefully acknowledges support from the Agence Nationale de la Recherche through Labex ACTION program (Contract No. ANR-11-LABX-0001-01) and OptiRoC project (Grant No. ANR-12-BS04-0001-011). B. K. and A.C. acknowledge support from the Burgundy Region. A. C. acknowledges the Japan Society for the Promotion of Science for awarding the International Research Fellowship and support from The Association of German Engineers (VDI). Part of the work described in Sec. II and Appendix A was supported by the Russian Science 
Foundation (Grant No. 14-22-00174 to V.Z. and A. G.). A. G. also acknowledges the support of the RFBR (Research Project No. 14-01-31378 mol_a) and RF President Fellowship program for young scientists (No. 2028.2015.5). V.Z. acknowledges National Science Foundation (No. 1130450). N. A. acknowledges the support of the Australian Research Council (Project No. DP140100265) and the Volkswagen Foundation.

\section{APPENDIX A: THEORETICAL TOOLS}

We find a general multibreather solution of the focusing NLSE by using a powerful integration technique-the dressing method. Integration of the focusing NLSE in the presence of a background plane wave requires the study of the auxiliary linear Zakharov-Shabat system [22] with the complex spectral parameter $\lambda=\lambda_{\mathrm{Re}}+i \lambda_{\mathrm{Im}}$. The solution of this system (wave function) has a cut on the real axes where $-A<\lambda_{\mathrm{Re}}<A$ (here, $A$ is amplitude of the background wave). Each breather adds a simple pole to the wave function at some point of the $\lambda$ plane, including possibly the cut (in this case, we obtain the Akhmediev breather). Coordinates of the pole in the $\lambda$ plane describe the main breather properties (amplitude, group velocity, period of oscillation, etc.). We use the Joukowsky transform, which maps the cut onto the circle of unit radius and the $\lambda$ plane onto the outer part of this circle, so that the coordinates of the pole are described by the radial and angle coordinates $R$ and $\alpha$. Furthermore, each breather has two additional parameters $\mu$ and $\theta$, which describe phase shifts in space and time and between different breathers.

Zakharov and Gelash have found that the cut in the $\lambda$ plane allows construction of such a configuration of poles (when the poles are paired near the cut on the opposite sides) that a corresponding solution must be a small perturbation of the initial plane wave at the moment of breather collision. These solutions were called superregular. The theoretical details can be found in Refs. [33,34]. Here, we briefly discuss how the simplest one-pair superregular solution [see Eq. (1)] can be obtained starting from the general two-breather solution. The general two-breather solution has four main parameters, $R_{1,2}, \alpha_{1,2}$, and four additional phase-shift parameters, $\mu_{1,2}, \theta_{1,2}$ (subscripts 1 and 2 correspond to the first and second breather). To construct a one-pair superregular breather, first, we have to demand the phases of the solution to be the same at $\pm \infty$ by the $\tau$ coordinate at any fixed $\xi$, since a small perturbation of the plane wave background cannot change the phase of the whole solution at infinity. This happens when breathers move in opposite directions and $\alpha_{1}=-\alpha_{2}=\alpha$. Interestingly, that in this case, if $R_{1}=R_{2}=1$, the solution is a pure plain wave. This means that two Akhmediev breathers with opposite values of angular parameter completely annihilate each other. As a result, when $R_{1}$ and $R_{2}$ are close to 1 , we observe quasiannihilation-two quasiAkhmediev breathers almost annihilate at the moment of collision with the formation of a small perturbation of the plane wave. Thus, the amplitude of the perturbation is controlled by the difference of $R_{1,2}$ from the unity. In general, it is not necessary to demand that $R_{1}=R_{2}=R$, but here we study only this simple case. Now, $\varepsilon=R-1$ can be considered as the small parameter in our theory. The last important step is adjusting the phase parameters $\mu_{1,2}, \theta_{1,2}$. In principle, annihilation can be observed at wide range of $\mu_{1,2}, \theta_{1,2}$; however, as we mention in Sec. II, the most effective annihilation occurs when $\theta_{1}+\theta_{2}$ is close to $\pi$.

The detailed description of the impact of parameters $\mu$ and $\theta$ can be found in Refs. [33,34]. Here, we only mention that $\theta$ corresponds to phase shifts between breathers and affects the shape of the perturbation. By changing $\mu$, we can shift the breather's collision in time and space from the point $(0,0)$ to the point $\xi_{0}=\left(\mu_{1}-\mu_{2}\right) / 4 \gamma, \tau_{0}=\left(\mu_{1}+\mu_{2}\right) / 4 \eta$, where $\gamma$ and $\eta$ are defined in Eq. (1). However, it should be noted that changing $\mu$ leads to the changing phase shifts between the breathers as well. That is why we measure different superregular perturbations in experiments with the growth of perturbation and annihilation of two quasiAkhmediev breathers.

\section{APPENDIX B: WATER WAVE TANK}

All experiments are conducted in deep-water conditions, meaning that the tanh of the product of the wave number $k_{0}=10 \mathrm{rad} \mathrm{m}^{-1}$ and of the water depth of $1 \mathrm{~m}$ is the unity. The single-flap wave-generating paddle is activated and controlled by a hydraulic cylinder and the flap displacement is checked to be proportional to the generated surface height. The water-surface elevation at any given point is measured by a capacitance wave gauge with a sensitivity of $1.06 \mathrm{~V} / \mathrm{cm}$ and a sampling frequency of $0.5 \mathrm{kHz}$. In order to excite the modulation instability in the tank, one has to fix the initial amplitude $a_{0}$ and the wave number of the carrier wave $k_{0}$. These two parameters define the steepness $a_{0} k_{0}$ of the quasiregular wave. The frequency of the carrier wave is given by the dispersion relation of linear deepwater wave theory, $\omega_{0}=\left(g k_{0}\right)^{1 / 2}$, where $g$ is the gravitational acceleration. The surface elevation $\eta(z, t)$ is related to the NLSE solution $\psi$ to second order in steepness as follows:

$\eta(z, t)=\operatorname{Re}\left\{\psi(z, t) e^{i\left(k_{0} z-\omega_{0} t\right)}+\frac{1}{2} k_{0} \psi^{2}(z, t) e^{2 i\left(k_{0} z-\omega_{0} t\right)}\right\}$

The correspondence between the theory and experiment can be retrieved by recalling that dimensional distance $z(\mathrm{~m})$ and time $t(\mathrm{~s})$ are related to the previous rescaled variables by $z=\tau /\left(\sqrt{2} k_{0}^{2} a_{0}\right)+c_{g} t$ and $t=2 \xi /\left(k_{0}^{2} a_{0}^{2} \omega_{0}\right)$, with $c_{g}=\omega_{0} /\left(2 k_{0}\right)$. Using these latter relations and inverting the scaling, the analytical solution, multiplied 
by the carrier amplitude $a_{0}$, is then written in dimensional form, satisfying the hydrodynamic NLSE [4], in order to define the exact initial conditions of modulation instability and then observe the expected wave dynamics at any desired location in the wave tank. This automatically provides the initial wave profile at the wave maker and consequently the signal that drives the paddle.

In Figs. 4(a) and 4(c), the initial conditions for the water surface are determined by Eq. (B1) evaluated at $z=0$ and for the carrier amplitude $a_{0}=0.01 \mathrm{~m}$ and steepness $a_{0} k_{0}=0.1$, while the breather parameters are $R=1.15$, $\alpha=0.4, \theta_{1,2}=\pi / 2+\pi, \mu_{1,2}=0$ and $R=1.15, \alpha=0.4$, $\theta_{1,2}=\pi / 2, \mu_{2,1}= \pm 2$, respectively. Note that we added $\pi$ in the initial values of $\theta_{1,2}$ due to our choice of physical scaling described above. At a specific distance, the temporal variation of the envelope can be easily reconstructed from the surface measurements, by use of the Hilbert transform [23]. Deviations can be attributed to higher-order effects that cannot be captured within the standard NLSE. As expected, the agreement becomes worse when nonlinearity is significantly increased during the breather compression process. An experimentally observed asymmetry can be explained in terms of higher-order NLS equations with odd terms such as the modified NLSE [51] or similar [52,53] equations that account for the formation of asymmetric wave packets.

\section{APPENDIX C: OPTICAL WORKBENCH}

The experimental setup shown in Fig. 3(b) is mainly based on high-speed telecommunications-grade components. The initial frequency comb is generated by the implementation of a $20-\mathrm{GHz}$ repetition rate pulse source centered at $1550 \mathrm{~nm}$ based on the nonlinear compression of an initial beat signal in a cavityless optical-fiber-based device. More details about a similar fiber-based frequency comb source can be found in Ref. [54]. The spectrum of such a pulse source can be approximated as a series of Dirac $\delta$ functions separated by the repetition rate; it is characterized by a typical triangular spectrum on a logarithmic scale whose spectral width depends on the nonlinear compression. Here, the source delivers nearly 3.3-ps pulses with $\sim 5-\mathrm{W}$ peak power. A programmable optical filter (wave shaper) provides an extremely fine control of the amplitude and phase characteristics of each line of the frequency comb. The high resolution $(\sim 1 \mathrm{GHz})$ of this solid-state liquid crystal on silicon system allows us to select or control individual spectral peaks of the comb. Relative intensity (over a 50-dB dynamic range) and phase differences of 24 sidebands with the central peak at $1550 \mathrm{~nm}$ (i.e., the background wave) are managed to define the exact initial conditions of modulation instability. An erbium-doped fiber amplifier is used to amplify and fix the average power of the synthesized wave according to the theoretical parameters (for each element of the periodic pattern) before coupling into a 0.76 - or $1.5-\mathrm{km}$-long segment of the same standard single-mode optical fiber (Corning SMF-28) with group velocity dispersion $\beta_{2}=-21.1 \mathrm{ps}^{2} \mathrm{~km}^{-1}$, linear losses $\alpha_{\mathrm{dB}}=0.2 \mathrm{~dB} \mathrm{~km}^{-1}$, and nonlinearity $\gamma=1.2 \mathrm{~W}^{-1} \mathrm{~km}^{-1}$. At the fiber output, the optical intensity profile is characterized using an ultrafast optical sampling oscilloscope with subpicosecond resolution and a high-dynamic-range optical spectrum analyzer with $2.5-\mathrm{GHz}$ resolution.

The correspondence between theory and experiment can be retrieved by recalling that the dimensional distance $z(\mathrm{~m})$ and time $t$ (s) are related to the previous normalized parameters by $z=\xi L_{\mathrm{NL}}$ and $t=\tau t_{0}$, where the characteristic (nonlinear) length and time scales are $L_{\mathrm{NL}}=(\gamma P 0)^{-1}$ and $t_{0}=\left(\left|\beta_{2}\right| L_{\mathrm{NL}}\right)^{1 / 2}$, respectively. The dimensional field $E(z, t)\left(\mathrm{W}^{1 / 2}\right)$ is $E=P_{0}^{1 / 2} \psi, P_{0}$ being the average power of the perturbed continuous wave, here equal to $0.74 \mathrm{~W}$. In Figs. 5 and 6, the initial conditions at the fiber input are $\psi\left(R=1.4, \alpha=0.4, \theta_{1,2}=\pi / 2, \mu_{2,1}= \pm 2, \xi=-1, \tau\right)$ and $\psi\left(R=1.4, \alpha=0.4, \theta_{1,2}=\pi / 2+\pi, \quad \mu_{2,1}= \pm 2\right.$, $\xi=-2.85, \tau)$, respectively. By calculating spectral (phase and intensity) profile differences between the initial frequency comb and the corresponding Fourier transform of the initial condition at the fiber input, one can apply the resulting (phase and intensity) corrections as a pulseshaping mask to the wave shaper. Measured experimental wave profiles in both time and frequency domains are almost indistinguishable from predictions in Figs. 5 and 6, since the cumulated fiber losses are quite low over the propagation distance studied here. In particular, fiber characteristics are chosen in accordance with both spectral bandwidth and peak power of the wave evolving into the fiber in order to avoid the potential impact of higher-order dispersive or nonlinear effects [55]. Numerical simulations based on the NLSE and the generalized nonlinear Schrödinger equation, known to provide an accurate description of the propagation of any optical wave in optical fibers [55], are initially performed and then compared to design the experimental test bed according to the theoretical parameters.

[1] P. L. Christiansen, M. P. Sørensen, and A. C. Scott, Nonlinear Science at the Dawn of the 21st Century (Springer, Berlin, 2000).

[2] N. Akhmediev and A. Ankiewicz, Solitons, Nonlinear Pulses and Beams (Chapman and Hall, London, 1997).

[3] M. Remoissenet, Waves Called Solitons: Concepts and Experiments (Springer, Heidelberg, 2010).

[4] V. E. Zakharov and A. B. Shabat, Exact Theory of TwoDimensional Self-Focusing and One-Dimensional SelfModulation of Waves in Nonlinear Media, Zh. Eksp. Teor. Fiz. 61, 118 (1971) [Sov. Phys. JETP 34, 62 (1972)].

[5] V.E. Zakharov, Stability of Periodic Waves of Finite Amplitude on a Surface of Deep Fluid, J. Appl. Mech. Tech. Phys. 9, 190 (1968); V. E. Zakharov and L. A. 
Ostrovsky, Modulation Instability: The Beginning, Physica (Amsterdam) 238D, 540 (2009).

[6] M. J. Lighthill, Contribution to the Theory of Waves in Non-Linear Dispersive Systems, J. Inst. Math. Appl. 1, 269 (1965).

[7] G. B. Whitham, A General Approach to Linear and Nonlinear Dispersive Waves Using a Lagrangian, J. Fluid Mech. 22, 273 (1965).

[8] V. I. Bespalov and V. J. Talanov, Filamentary Structure of Light Beams in Nonlinear Liquids, JETP Lett. 3, 307 (1966).

[9] T. B. Benjamin and J. E. Feir, The Disintegration of Wave Trains on Deep Water. Part 1: Theory, J. Fluid Mech. 27, 417 (1967).

[10] T. B. Benjamin, Instability of Periodic Wavetrains in Nonlinear Dispersive Systems, Proc. R. Soc. A 299, 59 (1967).

[11] T. Taniuti and H. Washimi, Self-Trapping and Instability of Hydromagnetic Waves along the Magnetic Field in a Cold Plasma, Phys. Rev. Lett. 21, 209 (1968).

[12] S. Watanabe, Self-Modulation of a Nonlinear Ion Wave Packet, J. Plasma Phys. 17, 487 (1977).

[13] H. Bailung and Y. Nakamura, Observation of Modulational Instability in a Multi-Component Plasma with Negative Ions, J. Plasma Phys. 50, 231 (1993).

[14] K. Tai, A. Hasegawa, and A. Tomita, Observation of Modulational Instability in Optical Fibers, Phys. Rev. Lett. 56, 135 (1986).

[15] P. Marquie, J. M. Bilbault, and M. Remoissenet, Generation of Envelope and Hole Solitons in an Experimental Transmission Line, Phys. Rev. E 49, 828 (1994).

[16] L. Salasnich, A. Parola, and L. Reatto, Modulational Instability and Complex Dynamics of Confined MatterWave Solitons, Phys. Rev. Lett. 91, 080405 (2003).

[17] E. Kuznetsov, Solitons in a Parametrically Unstable Plasma, Sov. Phys. Dokl. 22, 507 (1977).

[18] T. Kawata and H. Inoue, Inverse Scattering Method for the Nonlinear Evolution Equations under Nonvanishing Conditions, J. Phys. Soc. Jpn. 44, 1722 (1978).

[19] Y.C. Ma, The Perturbed Plane-Wave Solutions of the Cubic Schrödinger Equation, Stud. Appl. Math. 60, 43 (1979).

[20] D. H. Peregrine, Water Waves, Nonlinear Schrödinger Equations and Their Solutions, J. Aust. Math. Soc. Series B, Appl. Math. 25, 16 (1983).

[21] N. Akhmediev and V. I. Korneev, Modulation Instability and Periodic Solutions of the Nonlinear Schrödinger Equation, Theor. Math. Phys. 69, 1089 (1986).

[22] N. Akhmediev, V. M. Eleonskii, and N. E. Kulagin, Exact First-Order Solutions of the Nonlinear Schrödinger Equation, Theor. Math. Phys. 72, 809 (1987).

[23] A. R. Osborne, Nonlinear Ocean Waves and the Inverse Scattering Transform (Academic Press, San Diego, 2010).

[24] B. Kibler, J. Fatome, C. Finot, G. Millot, F. Dias, G. Genty, N. Akhmediev, and J. M. Dudley, The Peregrine Soliton in Nonlinear Fibre Optics, Nat. Phys. 6, 790 (2010).

[25] A. Chabchoub, N. P. Hoffmann, and N. Akhmediev, Rogue Wave Observation in a Water Wave Tank, Phys. Rev. Lett. 106, 204502 (2011).

[26] H. Bailung, S. K. Sharma, and Y. Nakamura, Observation of Peregrine Solitons in a Multicomponent Plasma with Negative Ions, Phys. Rev. Lett. 107, 255005 (2011).
[27] M. Erkintalo, K. Hammani, B. Kibler, C. Finot, N. Akhmediev, J. M. Dudley, and G. Genty, Higher Order Modulation Instability in Nonlinear Fiber Optics, Phys. Rev. Lett. 107, 253901 (2011).

[28] B. Kibler, J. Fatome, C. Finot, G. Millot, G. Genty, B. Wetzel, N. Akhmediev, F. Dias, and J. M. Dudley, Observation of Kuznetsov-Ma Soliton Dynamics in Optical Fibre, Sci. Rep. 2, 463 (2012).

[29] A. Chabchoub, N. Hoffmann, M. Onorato, and N. Akhmediev, Super Rogue Waves: Observation of a Higher-Order Breather in Water Waves, Phys. Rev. X 2, 011015 (2012).

[30] B. Frisquet, B. Kibler, and G. Millot, Collision of Akhmediev Breathers in Nonlinear Fiber Optics, Phys. Rev. X 3, 041032 (2013).

[31] A. Chabchoub, B. Kibler, J. M. Dudley, and N. Akhmediev, Hydrodynamics of Periodic Breathers, Phil. Trans. R. Soc. A 372, 20140005 (2014).

[32] P. Dubard and V. B. Matveev, Multi-Rogue Waves Solutions: From the NLS to the KP-I Equation, Nonlinearity 26, R93 (2013).

[33] V.E. Zakharov and A. A. Gelash, Nonlinear Stage of Modulation Instability, Phys. Rev. Lett. 111, 054101 (2013).

[34] A. A. Gelash and V. E. Zakharov, Superregular Solitonic Solutions: A Novel Scenario for the Nonlinear Stage of Modulation Instability, Nonlinearity 27, R1 (2014).

[35] M. Onorato, S. Residori, U. Bortolozzo, A. Montina, and F. T. Arecchi, Rogue Waves and Their Generating Mechanisms in Different Physical Contexts, Phys. Rep. 528, 47 (2013).

[36] J. M. Dudley, G. Genty, F. Dias, B. Kibler, and N. Akhmediev, Modulation Instability, Akhmediev Breathers and Continuous Wave Supercontinuum Generation, Opt. Express 17, 21497 (2009).

[37] M. Erkintalo, G. Genty, B. Wetzel, and J. M. Dudley, Akhmediev Breather Evolution in Optical Fiber for Realistic Initial Conditions, Phys. Lett. A 375, 2029 (2011).

[38] S. Toenger, T. Godin, C. Billet, F. Dias, M. Erkintalo, G. Genty, and J. M. Dudley, Emergent Rogue Wave Structures and Statistics in Spontaneous Modulation Instability, Sci. Rep. 5, 10380 (2015).

[39] M. Tajiri and Y. Watanabe, Breather Solutions to the Focusing Nonlinear Schrödinger Equation, Phys. Rev. E 57, 3510 (1998).

[40] N. Akhmediev, J. M. Soto-Crespo, and A. Ankiewicz, Extreme Waves that Appear from Nowhere: On the Nature of Rogue Waves, Phys. Lett. A 373, 2137 (2009).

[41] N. Akhmediev, J. M. Soto-Crespo, and A. Ankiewicz, How to Excite a Rogue Wave, Phys. Rev. A 80, 043818 (2009).

[42] E. A. Kuznetsov, Fermi-Pasta-Ulam Recurrence and Modulation Instability (to be published).

[43] N. Akhmediev, D. R. Heatley, G. I. Stegeman, and E. M. Wright, Pseudo-Recurrence in Two-Dimensional Modulation Instability with a Saturable Self-Focusing Nonlinearity, Phys. Rev. Lett. 65, 1423 (1990).

[44] N. Akhmediev, Déjà Vu in Optics, Nature (London) 413, 267 (2001).

[45] D. M. Henderson and H. Segur, The Role of Dissipation in the Evolution of Ocean Swell, J. Geophys. Res. 118, 5074 (2013). 
[46] A. Chowdury, A. Ankiewicz, and N. Akhmediev, Moving Breathers and Breather-to-Soliton Conversions for the Hirota Equation, Proc. R. Soc. A 471, 20150130 (2015).

[47] S. Coen and M. Haelterman, Modulational Instability Induced by Cavity Boundary Conditions in a Normally dispersive Optical Fiber, Phys. Rev. Lett. 79, 4139 (1997).

[48] M. Conforti, A. Mussot, J. Fatome, A. Picozzi, S. Pitois, C. Finot, M. Haelterman, B. Kibler, C. Michel, and G. Millot, Turbulent Dynamics of an Incoherently Pumped Passive Optical Fiber Cavity: Quasisolitons, Dispersive Waves, and Extreme Events, Phys. Rev. A 91, 023823 (2015).

[49] D. V. Churkin, S. Sugavanam, N. Tarasov, S. Khorev, S. V. Smirnov, S. M. Kobtsev, and S. K. Turitsyn, Stochasticity, Periodicity and Localized Light Structures in Partially Mode-Locked Fibre Lasers, Nat. Commun. 6, 7004 (2015).

[50] A. Chabchoub, B. Kibler, C. Finot, G. Millot, M. Onorato, J.M. Dudley, and A. V. Babanin, The Nonlinear
Schrödinger Equation and the Propagation of Weakly Nonlinear Waves in Optical Fibers and on the Water Surface, Ann. Phys. (Amsterdam) 361, 490 (2015).

[51] K. B. Dysthe, Note on the Modification of the Nonlinear Schrödinger Equation for Application to Deep Water Waves, Proc. R. Soc. A 369, 105 (1979).

[52] K. Trulsen and K. B. Dysthe, A Modified Nonlinear Schrödinger for Broader Bandwidth Gravity Waves on Deep Water, Wave Motion 24, 281 (1996).

[53] W. Craig, P. Guyenne, and C. Sulem, Hamiltonian Approach to Nonlinear Modulation of Surface Water Waves, Wave Motion 47, 552 (2010).

[54] B. Frisquet, A. Chabchoub, J. Fatome, C. Finot, B. Kibler, and G. Millot, Two-Stage Linear-Nonlinear Shaping of an Optical Frequency Comb as Rogue Nonlinear-SchrödingerEquation-Solution Generator, Phys. Rev. A 89, 023821 (2014).

[55] G. P. Agrawal, Nonlinear Fiber Optics, 5th ed. (Academic Press, Oxford, England, 2013). 